\title{
TRAJECTORY GENERATION FOR QUANTUM SYSTEMS BASED ON LYAPOUNOV TECHNIQUES
}

\author{
Mazyar Mirrahimi* Pierre Rouchon**
}

\author{
* Ecole des Mines de Paris, Centre Automatique et \\ Systèmes, 60 Bd Saint-Michel, 75272 Paris cedex 06, \\ FRANCE.Email: mazyar.mirrahimi@ensmp.fr \\ ** Ecole des Mines de Paris, Centre Automatique et \\ Systèmes, 60 Bd Saint-Michel, 75272 Paris cedex 06, \\ FRANCE.Email: pierre.rouchon@ensmp.fr
}

\begin{abstract}
The convergence of a Lyapounov based control of the Schrödinger equation (finite dimensional) is analyzed via Lasalle invariance principle. When the linear tangent approximation around the goal eigen-state is controllable, such a feedback ensures global asymptotic convergence. When this linear tangent system is not controllable, the stability of the closed-loop system is not asymptotic. To overcome such lack of convergence we propose a modification based on adiabatic invariance. Simulations illustrate the simplicity and also the interest of these Lyapounov based controls for trajectory generation. Such control methods can also be adapted to tracking.
\end{abstract}

Keywords: Nonlinear systems, quantum systems, Control Lyapounov function, LaSalle's invariant set, adiabatic invariant.

\section{INTRODUCTION}

Controllability of a finite dimensional quantum system:

$$
\imath \dot{\Psi}=\left(H_{0}+u(t) H_{1}\right) \Psi
$$

where $H_{0}$ and $H_{1}$ are $n \times n$ Hermitian matrices with coefficients in $\mathbb{C}$, can be studied via the general accessibility criteria proposed in (Sussmann and Jurdjevic, 1972) and based on Lie-Brackets. More specific results might be found in e.g. (Ramakrishna et al., 1995) and (Turinici and Rabitz, 2003). In particular, the system is controllable if and only if the Lie algebra generated by the skew-symmetric matrices $H_{0} / \imath$ and $H_{1} / \imath$ is $s u(n)$. Thus controllability of such systems is well characterized. However, such a characterization does not provide a simple and efficient way to generate trajectories between two different states.
Optimal control techniques (see, e.g., (Maday and Turinici, 2003) and the reference herein) provides a first set of methods to generate trajectories. Another set consisting in using inversion techniques such as in (Rabitz, 2003) where the control task is to transfer the population from the ground vibrational state to the first vibrational state in a Morse potential modeling the vibrational motion of $\mathrm{O}-\mathrm{H}$.

In the same perspective, we propose here another method for generating the trajectories based on Lyapounov based techniques and closely related to (Jurdjevic and Quinn, 1978). Exploiting linearity with respect to the state, we show here that we can fully analyze the convergence and the links between asymptotic stability of the closedloop system and its controllability. We completely characterize the largest invariant Lasalle subset. 
This yields to a very simple necessary and sufficient condition for global asymptotic convergence of the closed-loop system: the tangent linear system must be controllable around the goal eigenstate.

In section 2, we present the Lyapounov-based design and illustrate the method via simulations on two 3-states examples: for the first example, the closed-loop is asymptotically stable whereas for the second one it is stable but not asymptotically stable. In section 3, we perform the convergence analysis based on Lasalle invariance principle. Asymptotic and exponential convergence is shown to be equivalent to the controllability of the tangent system around the goal eigen-state. Section 4 deals with the degenerate case where the tangent is not controllable. We add to the control an adiabatic open-loop control. Simple arguments based on adiabatic invariance indicates that we can recover the asymptotic convergence of the closedloop system. Simulations on the second example confirms the interest of such simple modification of the basic feedback scheme.

The authors thank Gabriel Turinici for many interesting discussions and references.

\section{THE TIME-VARYING FEEDBACK}

\subsection{Lyapounov based design}

Consider the quantum system $(\hbar=1)$

$$
\imath \frac{d}{d t} \Psi=\left(H_{0}+u(t) H_{1}\right) \Psi
$$

where $H_{0}$ and $H_{1}$ are $n \times n$ Hermitian matrices with coefficients in $\mathbb{C}$. Here $H_{0}$ is a time independent Hamiltonian, corresponding to the free evolution of the system in the absence of any external fields. The external interaction here is taken as a control field amplitude $u(t) \in \mathbb{R}$ coupled to the system through the time independent Hamiltonian $H_{1}$. The wave function $\Psi=\left(\Psi_{i}\right)_{i=1}^{n}$ is a vector in $\mathbb{C}^{n}$, verifying the conservation of probability:

$$
\sum_{i=1}^{n}\left|\Psi_{i}\right|^{2}=1 .
$$

An important characteristic is that the choice of the global phase is arbitrary: physically, the probability amplitudes $\Psi$ and $e^{\imath \theta(t)} \Psi$ describe the same physical state for any global phase $t \mapsto$ $\theta(t) \in \mathbb{R}$.

The conservation of probability and global phase invariance have important consequences on the geometry of the physical state space: $\Psi$ lives on the unit sphere of $\mathbb{C}^{n}$; two probability amplitudes $\Psi_{1}$ and $\Psi_{2}$ are identified when exists $\theta \in \mathbb{R}$ such that $\Psi_{1}=\exp (\imath \theta) \Psi_{2}$. Thus the geometry of the state space does not coincides with the unit sphere of $\mathbb{C}^{n}$, i.e., $\mathbb{S}^{2 n-1}$.

The usual way to take into account such geometry is to reduce the dynamics on the minimal state space: when $n=2$, the dynamics reduces on $\mathbb{S}^{2}$, the unit sphere of $\mathbb{R}^{3}$ called the Bloch sphere. This corresponds to the geometric representation in terms of a fictitious spin 1/2 (see, e.g.,(CohenTannoudji et al., 1977)). For $n>2$ such reduction is less simple and the state-space geometry corresponds to the complex projective space of $\mathbb{C}^{n}$, $\mathbb{P} \mathbb{C}^{n}$.

In this paper, we propose another way to take into account such non trivial geometry of the physical state-space. Instead of reducing the state dimension, we increase the number of controls by one. To $u$ we will add a second control $\omega$ corresponding to the time derivative of the global phase. Thus we consider instead of (1) the following control system

$$
\imath \dot{\Psi}=\left(H_{0}+u H_{1}+\omega\right) \Psi
$$

where $\omega \in \mathbb{R}$ is a new control playing the role of a gauge degree of freedom. We can choose it arbitrarily without changing the physical quantities attached to $\Psi$. With such additional fictitious control $\omega$, we will assume in the sequel that the state space is $\mathbb{S}^{2 n-1}$ and the dynamics given by (3) admits two independent controls $u$ and $\omega$.

Remark 1. Adding controls to take into-account symmetry is not new. It has been already proposed for induction motors by Blaschke (Blaschke, 1972). This point has been re-explained in (Martin and Rouchon, 1998) and is widely used for induction motors (see e.g. (Espinosa and Ortega, 1995)).

Our goal is to steer the initial state $\Psi^{0}$ to a pure state associated to an eigen-vector $\phi \in \mathbb{C}^{n}$ of $H_{0}$ associated to the eigen-value (energy) $\lambda \in \mathbb{R}$. Thus we have $H_{0} \phi=\lambda \phi$ and $|\phi|=1$. Take the following real-value function $V(\Psi)$ :

$$
V(\Psi)=\langle\Psi-\phi \mid \Psi-\phi\rangle
$$

where $\langle. \mid$.$\rangle denotes the hermitian product. V$ is positive for all $\Psi \in \mathbb{C}^{n}$ and vanishes when $\Psi=\phi$. Simple computations show that $V$ is a control Lyapounov function:

$\frac{d}{d t} V=2 u \Im\left(\left\langle H_{1} \Psi(t) \mid \phi\right\rangle\right)+2(\omega+\lambda) \Im(\langle\Psi(t) \mid \phi\rangle)$

where $\Im$ denotes the imaginary part. By choosing $u$ and $\omega+\lambda$ with the opposite signs of $\Im\left(\left\langle H_{1} \Psi \mid \phi\right\rangle\right)$ and $\Im(\langle\Psi(t) \mid \phi\rangle)$ respectively, $V$ will decrease along the trajectories. Any feedback of the form 


$$
\begin{aligned}
& u=-a \Im\left(\left\langle H_{1} \Psi \mid \phi\right\rangle\right) \\
& \omega=-\lambda-b \Im(\langle\Psi \mid \phi\rangle) .
\end{aligned}
$$

where $a$ and $b$ are positive constants ensures $d V / d t \leq 0$ : with such feedback, the distance between the actual state $\Psi$ and the goal state $\phi$ decreases.

\subsection{Tutorial examples and simulations}
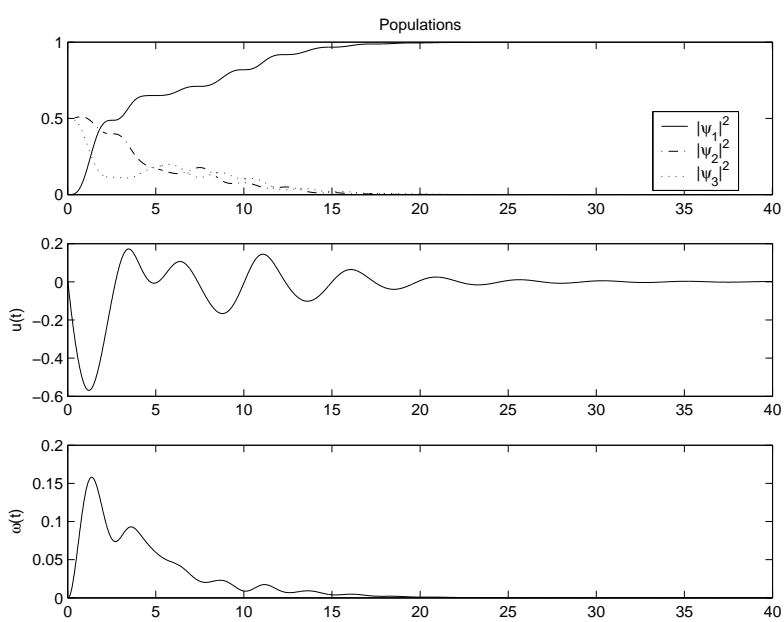

Fig. 1. populations $\left(\left(\left|\Psi_{1}\right|^{2},\left|\Psi_{2}\right|^{2},\left|\Psi_{3}\right|^{2}\right)\right.$ and controls $u$ and $\omega$; initial condition $(0,1 / \sqrt{2}, 1 / \sqrt{2}) ;$ system defined by $(7)$ with feedback (8).

Take $n=3, \Psi=\left(\Psi_{1}, \Psi_{2}, \Psi_{3}\right)^{T}$ and

$$
H_{0}=\left(\begin{array}{lll}
0 & 0 & 0 \\
0 & 1 & 0 \\
0 & 0 & \frac{3}{2}
\end{array}\right), \quad H_{1}=\left(\begin{array}{lll}
0 & 1 & 1 \\
1 & 0 & 1 \\
1 & 1 & 0
\end{array}\right)
$$

Let us use the previous Lyapunov control in order to trap our system in the first eigen-state $\phi=$ $(1,0,0)$ of energy $\lambda=0$. We have (* means complex conjugate)

$$
\Im\left(\left\langle H_{1} \Psi \mid \phi\right\rangle\right)=\Im\left(\Psi_{2}^{\star}+\Psi_{3}^{\star}\right)
$$

and we take (6) with $a=b=1$ :

$$
\begin{aligned}
& u=-\frac{1}{2} \Im\left(\Psi_{2}^{\star}+\Psi_{3}^{\star}\right) \\
& \omega=-\frac{1}{2} \Im\left(\Psi_{1}^{\star}\right)
\end{aligned}
$$

Simulations of Figure 1 describes the trajectory with $\Psi^{0}=(0,1 / \sqrt{2}, 1 / \sqrt{2})$ as initial state. Other simulations indicate that the trajectories always converges to $\phi$. It appears that such Lyapunov based technics is quite efficient for system (7). In Theorem 2, it is shown that almost global convergence is equivalent to the controllability of the linear tangent system around $\phi$.

Let us consider another example that clearly illustrates the basic limitation of such Lyapunov
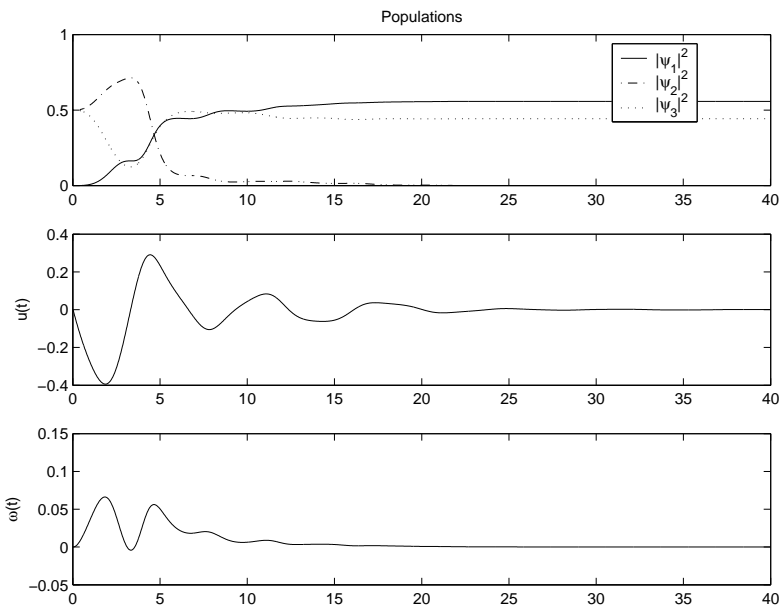

Fig. 2. populations $\left(\left(\left|\Psi_{1}\right|^{2},\left|\Psi_{2}\right|^{2},\left|\Psi_{3}\right|^{2}\right)\right.$ and controls $u$ and $\omega$; initial condition $(0,1 / \sqrt{2}, 1 / \sqrt{2}) ; \quad H_{0}$ defined by $(7), \quad H_{1}$ by (9)and feedback by (10).

based technique: $H_{0}$ and the goal state $\phi$ remain unchanged but $H_{1}$ becomes:

$$
H_{1}=\left(\begin{array}{lll}
0 & 1 & 0 \\
1 & 0 & 1 \\
0 & 1 & 0
\end{array}\right)
$$

The feedback becomes

$$
u=-\frac{1}{2} \Im\left(\Psi_{2}^{\star}\right), \quad \omega=-\frac{1}{2} \Im\left(\Psi_{1}^{\star}\right) .
$$

Simulations of Figure 2 start with $(0,1 / \sqrt{2}, 1 / \sqrt{2})$ as initial condition for $\Psi$. We clearly realize that such a feedback reduces the distance with the first state but does not ensure its convergence to 0 . This is not due to a lack of controllability. This system is controllable since the Lie algebra spanned by $H_{0} / \imath$ and $H_{1} / \imath$ coincides with su(3) (Ramakrishna et al., 1995). As explained in Theorem 2, such convergence deficiency comes form the fact that the linear tangent system around $\phi$ is not controllable.

\section{CONVERGENCE ANALYSIS}

The goal of this section is to prove the following theorem that underlies simulations of figures 1 and 2 .

Theorem 2. Consider (3) with $\Psi \in \mathbb{S}^{2 n-1}$ and an eigen-state $\phi \in \mathbb{S}^{2 n-1}$ of $H_{0}$ associated to the eigenvalue $\lambda$. Take the static feedback (6) with $a, b>0$. Then the two following propositions are true:

(1) If the spectrum of $H_{0}$ is not degenerate (all eigen-values are distinct), the $\Omega$-limit set of the closed loop system is the intersection of $\mathbb{S}^{2 n-1}$ with the real vector-space $E$ spanned by the eigen-vectors $\Phi$ of $H_{0}$ such that $\left\langle H_{1} \Phi \mid \phi\right\rangle=0$ and $\Im(\langle\Phi \mid \phi\rangle)=0$. 
(2) The $\Omega$-limit set reduces to $\{\phi,-\phi\}$ if and only if $H_{0}$ is not degenerate and $E=\mathbb{R} \phi$. In this case: the equilibrium $\phi$ is exponentially stable (on $\mathbb{S}^{2 n-1}$ ); the equilibrium $-\phi$ is unstable; the attractor set of $\phi$ is exactly $\mathbb{S}^{2 n-1} /\{-\phi\}$. This case corresponds to the controllability of the linear-tangent system at $\phi$, a time-invariant linear system that lives on the $2 n-1$ plane tangent to $\mathbb{S}^{2 n-1}$ at $\phi$.

For example of Figure 1, it becomes clear that $E=$ $\mathbb{R} \phi$ since $H_{0}$ is not degenerate and $\phi=(1,0,0)$ is almost globally asymptotically stable. Notice the condition $E=\mathbb{R} \phi$ says that, physically, the goalstate $\phi$ is connected to all other excited states via mono-photonic transition (see, e.g., (Messiah, 1962)).

For example of Figure 2, element of $E$ are of the form $(x, 0, z)$ where $x \in \mathbb{R}$ and $z \in \mathbb{C}$; we observe effectively that the $\Omega$-limit set contains elements of the form $(x, 0, r \exp (\imath \theta))$ with $x, r$ and $\theta$ in $\mathbb{R}$ such that $x^{2}+r^{2}=1$. Physically, the transition between $\phi$ and state of energy $3 / 2$ necessitates at least two photons: the feedback (10) cannot find such multi-photonic processes.

The proof of Theorem 2 mainly relies on the characterization of the $\Omega$-limit set via Lasalle invariance principle. It provides here a complete description of the invariant subset via the linear system $M \Xi=0$ where $M$ is defined by (12) here below. Such description becomes very simple when $H_{0}$ is not degenerate.

\subsection{Proof of proposition (1) of Theorem 2}

Up to a shift on $\omega$ and $H_{0}$, we can assume that $\lambda=$ 0 . Lasalle's principle (see, e.g., theorem 3.4, page 115 of (Khalil, 1992)) says that the trajectories of the closed-loop system converge to the largest invariant set contained in $d V / d t=0$ where $V$ is defined by (4). The equation $d V / d t=0$ means that

$$
\Im\left(\left\langle H_{1} \Psi \mid \phi\right\rangle\right)=\Im(\langle\Psi \mid \phi\rangle)=0,
$$

Thus $u=0$ and $\omega=0$. Invariance means that with $\imath \frac{d}{d t} \Psi=H_{0} \Psi, \frac{d}{d t} \Im\left(\left\langle H_{1} \Psi \mid \phi\right\rangle\right)=0$ and $\frac{d}{d t} \Im(\langle\Psi \mid \phi\rangle)=0$. Clearly $\frac{d}{d t} \Im(\langle\Psi \mid \phi\rangle)=0$ does not give any additional information since $H_{0} \phi=0$. Only $\frac{d}{d t} \Im\left(\left\langle H_{1} \Psi \mid \phi\right\rangle\right)=0$ provides a new independent equation: $\Re\left(\left\langle H_{1} H_{0} \Psi \mid \phi\right\rangle\right)=0$ that reads

$$
\Re\left(\left\langle\left[H_{0}, H_{1}\right] \Psi \mid \phi\right\rangle\right)=0 .
$$

Similarly $\frac{d}{d t} \Re\left(\left\langle\left[H_{0}, H_{1}\right] \Psi \mid \phi\right\rangle\right)=0$ implies

$$
\Im\left(\left\langle\left[H_{0},\left[H_{0}, H_{1}\right]\right] \Psi \mid \phi\right\rangle\right)=0 .
$$

And so on. Finally, the largest invariant set is characterized by $\Im(\langle\Psi \mid \phi\rangle)=0$ with the following conditions,

$$
\begin{aligned}
& \Im\left(\left\langle H_{1} \Psi \mid \phi\right\rangle\right)=0 \\
& \Re\left(\left\langle\left[H_{0}, H_{1}\right] \Psi \mid \phi\right\rangle\right)=0 \\
& \Im\left(\left\langle\left[H_{0},\left[H_{0}, H_{1}\right]\right] \Psi \mid \phi\right\rangle\right)=0 \\
& \ldots
\end{aligned}
$$

that corresponds to the "ad-conditions" obtained in (Jurdjevic and Quinn, 1978). At each step, we have the Lie bracket of the Hamiltonian $H_{0}$ with the Hamiltonian of the last step.

We can always assume that $H_{0}$ is diagonal. Then we can easily compute the commutator $\left[H_{0}, B\right]$ where $B=\left(B_{i j}\right)$ is a $n \times n$ matrix. With $H_{0}=$ $\operatorname{diag}\left(\lambda_{1}, \ldots, \lambda_{n}\right)$, we have

$$
\left[H_{0}, A\right]_{i, j}=\left(\lambda_{i}-\lambda_{j}\right) B_{i j} .
$$

Let take $B=H_{1}$ in order to simplify the notations. So:

$$
\begin{aligned}
{\left[H_{0}, B\right] } & =\left(\left(\lambda_{i}-\lambda_{j}\right) B_{i j}\right) \\
{\left[H_{0},\left[H_{0}, B\right]\right] } & =\left(\left(\lambda_{i}-\lambda_{j}\right)^{2} B_{i j}\right) \\
\vdots & \\
\underbrace{\left[H_{0},\left[H_{0}, \ldots,\left[H_{0}\right.\right.\right.}_{k \text { times }}, B]] \ldots] & =\left(\left(\lambda_{i}-\lambda_{j}\right)^{k} B_{i j}\right) .
\end{aligned}
$$

Thus the previous system reads:

$$
\begin{aligned}
& \Im\left(\Sigma_{j} B_{1 j} \Psi_{j}\right)=0, \\
& \Re\left(\Sigma_{j}\left(\lambda_{1}-\lambda_{j}\right) B_{1 j} \Psi_{j}\right)=0, \\
& \vdots \\
& \Im\left(\Sigma_{j}\left(\lambda_{1}-\lambda_{j}\right)^{2 k} B_{1 j} \Psi_{j}\right)=0, \\
& \Re\left(\Sigma_{j}\left(\lambda_{1}-\lambda_{j}\right)^{2 k+1} B_{1 j} \Psi_{j}\right)=0, \\
& \quad \ldots
\end{aligned}
$$

Set

$$
M=\left(\begin{array}{ccc}
1 & \cdots & 1 \\
\left(\lambda_{1}-\lambda_{2}\right)^{2} & \cdots & \left(\lambda_{1}-\lambda_{n}\right)^{2} \\
\left(\lambda_{1}-\lambda_{2}\right)^{4} & \cdots & \left(\lambda_{1}-\lambda_{n}\right)^{4} \\
\vdots & \vdots & \vdots \\
\left(\lambda_{1}-\lambda_{2}\right)^{2(n-2)} & \cdots & \left(\lambda_{1}-\lambda_{n}\right)^{2(n-2)}
\end{array}\right)
$$

and

$\Xi=\left(\left(1+\imath\left(\lambda_{1}-\lambda_{2}\right)\right) B_{12} \Psi_{2}, \ldots,\left(1+\imath\left(\lambda_{1}-\lambda_{n}\right)\right) B_{1 n} \Psi_{n}\right)^{T}$.

Then (11) implies that $M \Xi=0$. Indeed even if (11) corresponds to an infinite number of linear relations, using the Vandermonde structure of these equations one can easily see that it suffices to consider its first $n-1$ equations: higher order equations are necessarily linear combinations of the first $n-1$ equations. Thus the linear system $M \Xi=0$ with $\Im(\langle\Psi \mid \phi\rangle)=0$ provides a complete characterization of the $\Omega$-limit set.

In the particular case where the free Hamiltonian $H_{0}$ has a non-degenerate spectrum, $M$ is invertible and thus $\Xi=0$. Then $\Psi \in \mathbb{S}^{2 n-1}$ is in the $\Omega$-limit set if and only if

$$
B_{1 j} \Psi_{j}=0, \forall j \in\{2, \ldots, n\} .
$$

and $\Im\left(\Psi_{1}\right)=0$. 


\subsection{Proof of proposition (2) of Theorem 2}

Notice first that in any case the $\Omega$-limit set contains $\phi$ and $-\phi$.

If $H_{0}$ has a non-degenerate spectrum and $E=\mathbb{R} \phi$ then proposition (1) implies that the $\Omega$-limit set is just $\{ \pm \phi\}$. Now let us suppose that at least one of these two conditions is not fulfilled.

Assume that $E \neq \mathbb{R} \phi$. Thus exists an eigen-vector $\Phi$ of $H_{0}$ not co-linear to $\phi$ such that $\left\langle H_{1} \Phi \mid \phi\right\rangle=0$. With $\Psi(0)=\Phi$ as initial state, we have $u(t)=0$ and $\omega(t)=-\lambda$ and $\Psi(t)=\Phi$ for all $t>0$. The $\Omega$-limit set contains $\Phi$.

Assume $E=\mathbb{R} \phi$ but that $H_{0}$ has a degenerate spectrum. We will consider two cases:

(1) There exists an eigen-vector $\phi_{k}$ with length 1 of $H_{0}$ orthogonal to $\phi$ but with the same eigen-value $\lambda$. Since $E=\mathbb{R} \phi, B_{1 k}=$ $\left.\left\langle H_{1} \phi_{k} \mid \phi\right\rangle\right) \neq 0$. With $\Psi(0)=\frac{B_{1 k}}{\left|B_{1 k}\right|} \phi_{k}$ as initial state, we have $u(t)=0, \omega=-\lambda$ and $\Psi(t)=\frac{B_{1 k}}{\left|B_{1 k}\right|} \phi_{k}$ belongs to the $\Omega$-limit set.

(2) There exist two orthogonal eigen-vectors $\phi_{k}$ and $\phi_{l}$ of $H_{0}$, with length one and admitting the same eigen-value $\mu \neq \lambda$. Since $E=$ $\left.\mathbb{R} \phi, B_{1 k}=\left\langle H_{1} \phi_{k} \mid \phi\right\rangle\right) \neq 0$ and $B_{1 l}=$ $\left.\left\langle H_{1} \phi_{l} \mid \phi\right\rangle\right) \neq 0$. With $\Psi(0)=\left(B_{1 k} \phi_{l}-\right.$ $\left.B_{1 l} \phi_{k}\right) / \sqrt{\left|B_{1 k}\right|^{2}+\left|B_{1 l}\right|^{2}}$, we have $u(t)=0$, $\omega=-\lambda$ and

$$
\Psi(t)=e^{-\imath(\mu-\lambda) t} \Psi(0) .
$$

Thus the $\Omega$-limit set contains $\left(e^{\imath \alpha} \Psi(0)\right)_{\alpha \in[0,2 \pi]}$.

The proof of the first part of proposition (2) is thus done.

Let us prove now that $\phi$ is locally exponentially stable when $H_{0}$ is not degenerate and $E=\mathbb{R} \phi$. We will prove that the linear tangent closedloop system is asymptotically stable. This will automatically implies that the equilibrium $\phi$ is hyperbolically stable. Set

$$
\Psi(t)=\phi+\Delta \Psi(t)
$$

with $\Delta \Psi$ small. Then up to second order terms we have

$$
\begin{aligned}
& \imath \frac{d}{d t} \Delta \Psi=\left(H_{0}-\lambda I\right) \Delta \Psi-a \Im\left(\left\langle H_{1} \Delta \Psi \mid \phi\right\rangle\right) H_{1} \phi \\
& \quad-b \Im(\langle\Delta \Psi \mid \phi\rangle) \phi
\end{aligned}
$$

and $\Re(\langle\Delta \Psi \mid \phi\rangle)=0$ (definition of the tangent space at $\phi$ to the unit sphere $\left.\mathbb{S}^{2 n-1}\right)$. Set $W(\Delta \Psi)=\frac{1}{2}\langle\Delta \Psi \mid \Delta \Psi\rangle$. Simple computations show that $d W / d t \leq 0$ and $E=\mathbb{R} \phi$ implies that the LaSalle's invariant set of this linearized system reduces to $\Delta \Psi=0$ on the tangent space at $\phi$ to $\mathbb{S}^{2 n-1}$.

The fact that $-\phi$ is unstable results from the fact that the Lyapunov function $V$ reaches 4 its maximum on $\mathbb{S}^{2 n-1}$ only for $\Psi=-\phi$. Thus if $\Psi(0) \neq-\phi$, then necessary $\Psi(t)$ must converge to the other point of the $\Omega$-limit set. Thus $\lim _{t \mapsto+\infty} \Psi(t)=\phi$; the equilibrium $-\phi$ is unstable, the attraction region of $\phi$ is $\mathbb{S}^{2 n-1} /\{-\phi\}$.

Let us finally proved that $H_{0}$ non degenerate and $E=\mathbb{R} \phi$ is equivalent to the controllability of the linear-tangent system at $\phi$.

Set $\Psi(t)=\phi+\Delta \Psi(t)$ with $\Re(\langle\Delta \Psi \mid \phi\rangle)=0$, $u=\Delta u$ and $\omega=-\lambda+\Delta \omega$ with $\Delta \Psi, \Delta u$ and $\Delta \omega$ small. Then up to second order terms, (3) reads

$$
\imath \frac{d}{d t} \Delta \Psi=\left(H_{0}-\lambda I\right) \Delta \Psi+\Delta u H_{1} \phi+\Delta \omega \phi .
$$

Take $\left(\phi_{1}, \ldots, \phi_{n}\right)$ an ortho-normal eigen-basis of $H_{0}$ associated to $\left(\lambda_{1}, \ldots, \lambda_{n}\right)$ with $\phi_{1}=\phi$ and $\lambda_{1}=\lambda$. Set $\left(z_{1}, \ldots, z_{n}\right) \in \mathbb{C}^{n}$ the coordinates of $\Delta \Psi$ in this basis. Then: $\Re\left(z_{1}\right)=0$ and

$$
\begin{aligned}
\frac{d}{d t}\left(\Im\left(z_{1}\right)\right) & =-\Delta \omega-B_{11} \Delta u \\
\imath \frac{d}{d t} z_{2} & =\left(\lambda_{2}-\lambda_{1}\right) z_{2}+B_{12} \Delta u \\
\vdots & \\
\imath \frac{d}{d t} z_{n} & =\left(\lambda_{n}-\lambda_{1}\right) z_{n}+B_{1 n} \Delta u
\end{aligned}
$$

where $B_{i j}=\left\langle\phi_{i} \mid H_{1} \phi_{j}\right\rangle$. Controllability is then equivalent to the fact that $B_{1 i} \neq 0$ and $\lambda_{i} \neq \lambda_{j}$ for $i \neq j$ (use, e.g., Kalman controllability matrix). This is clearly equivalent to $H_{0}$ non degenerate and $E=\mathbb{R} \phi$.

\section{AN IDEA TO IMPROVE CONVERGENCE}

Theorem 2 and simulation of Figure 2 show that such Lyapounov method fails to steer the state $\Psi$ to the pure state associated to $\phi$ when the dimension of vector space $E$ exceeds 1 . However, the three states system of Figure 2 is controllable: the Lie algebra spanned by $H_{0} / \imath$ and $H_{1} / \imath$ is $s u(3)$.

To overcome such lack of convergence observed with simulations on figure 2, we will try to track an adiabatic reference trajectory

$$
\imath \frac{d}{d t} \Psi_{r}=\left(H_{0}+u_{r}(t) H_{1}\right) \Psi_{r}, \quad \Psi_{r}(0)=(1,0,0),
$$

where $u_{r}=\frac{1}{2} \sin (2 \pi t / T)$ with a period $T=300$, large compared with the natural periods of $H_{0}$ to ensure that $u_{r}$ is a slowly varying time function. Take the following tracking feedback

$u=u_{r}-\frac{1}{2} \Im\left(\left\langle H_{1} \Psi \mid \Psi_{r}(t)\right\rangle\right), \quad \omega=-\frac{1}{2} \Im\left(\left\langle\Psi \mid \Psi_{r}(t)\right\rangle\right)$.

Since $u_{r}$ varies slowly, adiabatic theory ensures that $\Psi_{r}$ will follow closely the first eigen-state of $H_{0}+u_{r} H_{1}$ (Messiah, 1962). So when $u_{r}$ returns to $0, \Psi_{r}$ will almost return to the first eigenspace spanned by $(1,0,0)$ : we have $\Psi_{r}(T) \approx$ 
$(\exp (\imath \theta), 0,0)$ for some phase shift $\theta$. If during this slow motion, the reference trajectory $\Psi_{r}$ is in the neighborhood of eigen-states of $H_{0}+u_{r} H_{1}$ where the linear tangent system is controllable, this will strongly improve convergence. This is effectively the case as shown in figure 3 that illustrates the efficiency of combining Lyapunov design and adiabatic invariance. Rigorous proofs of such convergence can be performed and will be given in future publications. See also (Beauchard et al., 2004) for a different method based on an implicitly defined control-Lyapunov function that ensures convergence then the linear-tangent system around $\phi$ is not controllable.

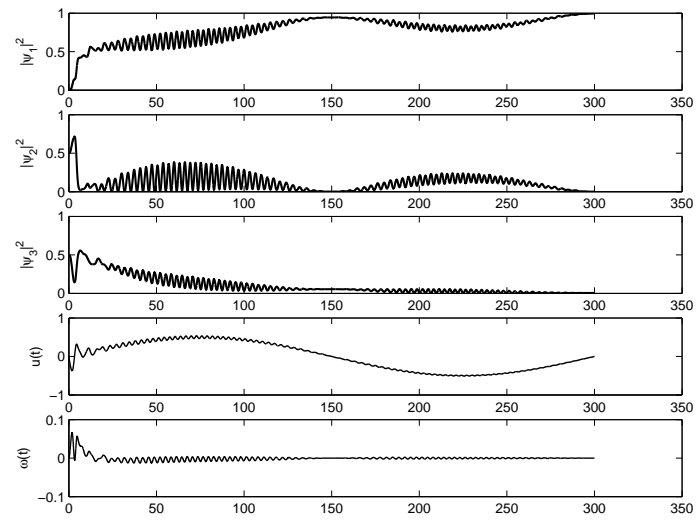

Fig. 3. system and initial conditions identical to figure 2; adiabatic trajectory (13) tracking via the feedback (14).

\section{CONCLUSION}

The convergence analysis presented here can be extended to trajectory tracking around generic reference trajectories. Consider a reference trajectory $t \mapsto\left(\Psi_{r}(t), u_{r}(t), \omega_{r}(t)\right)$ of $(1)$. Set $\Delta \Psi=$ $\Psi-\Psi_{r}, \Delta \omega=\omega-\omega_{r}$ and $\Delta u=u-u_{r}$. Then

$\imath \frac{d}{d t} \Delta \Psi=\left(H_{0}+u_{r}(t) H_{1}+\omega_{r}\right) \Delta \Psi+\Delta u H_{1} \Psi+\Delta \omega \Psi$.

Set $V=\langle\Delta \Psi \mid \Delta \Psi\rangle$. Simple computations yield $\frac{d}{d t} V=2 \Delta u \Im\left(\left\langle H_{1} \Psi(t) \mid \Psi_{r}\right\rangle\right)+2 \Delta \omega \Im\left(\left\langle\Psi(t) \mid \Psi_{r}\right\rangle\right)$. Thus any feedback where $\Delta u$ and $\Delta \omega$ are of the opposite sign with respect to $\Im\left(\left\langle H_{1} \Psi(t) \mid \Psi_{r}\right\rangle\right)$ and $\Im\left(\left\langle\Psi(t) \mid \Psi_{r}\right\rangle\right)$ respectively, ensures $\frac{d}{d t} V \leq 0$. A careful convergence analysis based on Lasalle invariant principle shows that, when the first variation around $\Psi_{r}$ is controllable, such simple feedback provides asymptotic tracking. This point is developed in (Mirrahimi and Rouchon, 2004).

\section{REFERENCES}

Beauchard, K., J.-M. Coron, M. Mirrahimi and P. Rouchon (2004). Stabilization of a finite dimensional Schrödinger equation. Submitted to System and Control Letters.
Blaschke, F. (1972). The principle of field oriention as applied to the new Transvector closed loop control system for rotating field machines. Siemens review p. 217.

Cohen-Tannoudji, C., B. Diu and F. Laloë (1977). Mécanique Quantique. Vol. I\& II. Hermann, Paris.

Espinosa, G. and R. Ortega (1995). An output feedback globally stable controller for induction motors. IEEE Transactions on Automatic Control 40(1), 138-143.

Jurdjevic, V. and J.P. Quinn (1978). Controllability and stability. Journal of Differential Equations 28, 381-389.

Khalil, H.K. (1992). Nonlinear Systems. MacMillan.

Maday, Y. and G. Turinici (2003). New formulations of monotonically convergent quantum control algorithms. J. Chem. Phys.

Martin, Ph. and P. Rouchon (1998). Symmetry and field-oriented control of induction motors. Technical Report 493. Ecole des Mines de Paris, Centre Automatique et Systèmes.

Messiah, A. (1962). Quantum Mechanics. Vol. I\&II. North Holland Publ. Co.. Amsterdam.

Mirrahimi, M. and P. Rouchon (2004). Trajectory tracking for quantum systems: a lyapounov approach.. In: Proc. of the International Symposium MTNS'2004.

Rabitz, H. anf Zhu, W. (2003). Quantum control design via adaptive tracking. J. Chem. Phys.

Ramakrishna, V., M. Salapaka, M. Dahleh and H. Rabitz (1995). Controllability of molecular systems. Phys. Rev. A 51(2), 960-966.

Sussmann, H.J. and V. Jurdjevic (1972). Controllability of nonlinear systems. J. Differential Equations 12, 95-116.

Turinici, G. and H. Rabitz (2003). Wavefunction controllability in quantum systems. J. Phys. A 36, 2565-2576. 